\title{
Skill-biased technological change, endogenous labor supply and growth: A model and calibration to Poland and the US
}

\author{
Guido Cazzavillan ${ }^{\mathrm{a}, 1}$, Krzysztof Olszewski ${ }^{\mathrm{b}, *}$ \\ a University Ca' Foscari of Venice, Department of Economics, Cannaregio 873, Fondamenta S.Giobbe 30121 Venezia, Italy \\ ${ }^{\mathrm{b}}$ National Bank of Poland, Świętokrzyska Street 11/21, 00-919 Warszawa, Poland
}

\section{A R T I C L E I N F O}

\section{Keywords:}

Skill-biased technological change

Endogenous labor supply

Transition Economies

\begin{abstract}
A B S T R A C T
In this paper skill-biased technological change is linked with endogenous labor supply in a growth model. Elastic labor supply allows us to explain how the observed increasing unemployment of unskilled workers is caused by skill-biased technological change. Using empirical data on wages and education, we construct the time series for the skill-biased technology for Poland and the US. The empirical relevance of the model is tested by calibrating it to empirical data for Poland over the period 1996-2006 and for the US over the period 1992-2008. Our numerical analysis shows that if the skill-biased technological change is not followed by the growth of total factor productivity, then output, physical capital stock and consumption decline. With only two necessary inputs, namely the share of skilled workers in total population and the technology adopted by firms, this model allows to simulate the future behavior of the labor market.
\end{abstract}

(C) 2011 University of Venice. Published by Elsevier Ltd. All rights reserved.

\section{Introduction}

Skill-biased technological change, which has led to a strong increase of the skill premium and sometimes also to the rise in unemployment of unskilled workers, is debated extensively in the economic literature. ${ }^{2}$ The phenomenon of increased non-production worker share in the wage bill in OECD countries in the context of skill-biased technological change (SBTC) was studied among others by Machin and Van Reenen (1998) and Berman et al. (1998). Berman et al. (1998, p. 1246) found that "SBTC was pervasive over the past two decades, occurring simultaneously in most, if not in all, developed countries". This change occurred in the US as well as in other developed countries and the most affected industries were the skillintensive ones, namely machinery, electrical machinery and printing and publishing (Berman et al., 1998). They state that less skilled workers sometimes also face increased unemployment. A very similar pattern can be observed in Transition Economies, which also face skill-biased technological change and increasing unemployment of unskilled workers. ${ }^{3}$

Even though that skill-biased technological change has an impact on unemployment of the unskilled workers, the literature usually assumes that labor supply is inelastic. However, Machin and Van Reenen (1998, p. 1217) state: "We cannot

\footnotetext{
* Corresponding author.

E-mail address: krzysztof.olszewski@nbp.pl (K. Olszewski).

1 The views expressed in this paper are those of the authors and do not necessarily reflect the opinions of the National Bank of Poland.

2 A review of the literature can be found in Acemoglu (2002) and Machin (2008). Esposito and Steher (2007) provide a literature overview focusing on Transition Economies.

3 Focusing on the period 1995-2003 and using data at the 14 industries level (DA-DN code) Esposito and Steher (2007) find evidence of SBTC in Transition Economies. However, there is a sector bias as SBTC was concentrated in the skill-intensive and medium skill industries in Hungary and Poland. Another study, performed by Lorentowicz et al. (2005) at a higher disaggregation level (23 ISIC industries) in the period 1994-2002 links FDI with an increase of skilled labor share and wage inequality in Poland.
} 
deduce the full effect of technology on labor market structure without also closing the model by looking at the supply side effects and the non-manufacturing sector, which is beyond the scope of this paper".

We aim to enrich the literature by including the SBTC process in a growth model with endogenous labor supply and exogenous neutral technological change. Further on, we include the exogenously changing share of skilled workers in the production function. We model a household which consists of skilled and unskilled workers, who optimally choose how much to work and how much leisure to have. Nobody gets unemployed in the neoclassical sense and household members share the income and do not suffer when they do not work. Under the assumption of elastic labor supply our model explains the observed decreasing labor supply of unskilled workers and links it to skill-biased technological change. The increasing skill premium makes it less valuable for the unskilled to work. We calibrate the model and test its empirical relevance using data for Poland and for the US. Poland made a fast transition towards a market economy and it is the largest economy in Central and Eastern Europe. The US are the most developed economy.

The paper is organized as follows. We model the production function with skilled and unskilled labor and solve the household labor supply problem in Section 2. In Section 3 we provide empirical evidence for skill-biased technological change and calculate its time series for Poland and the US. In Section 4 the model is calibrated and a numerical analysis is performed. This allows to test the model's empirical relevance. Section 5 concludes the paper.

\section{The model}

We consider an economy populated by an infinite number of households and firms. The economy is perfectly competitive and everybody is a price taker. Households consist of skilled and unskilled workers who provide a fraction of their time to the production of a representative good. Throughout the paper skill-biased technological change and total factor productivity growth are strictly exogenous.

\subsection{The productive sector}

We set up the productive sector based on the specification of Caselli and Coleman (2006):

$$
y=k^{\alpha}\left[\left(A_{u} L_{u}\right)^{\sigma}+\left(A_{s} L_{s}\right)^{\sigma}\right]^{(1-\alpha) / \sigma} .
$$

Here $L_{u}$ is unskilled labor and $L_{s}$ is skilled labor. The elasticity of substitution between skilled and unskilled workers is $\epsilon=1 /(1-\sigma)$ and lies in the range $(1,2)$ (see Autor et al., 1998).

In addition to the Caselli and Coleman (2006) model, we allow the workers to decide how much labor they supply and put the model in a growth framework. The households live infinitely. Each household consists of an exogenously given fraction $u$ of unskilled workers and a fraction $1-u$ of skilled workers. Each type of worker is endowed with one unit of time. The unskilled (skilled) worker devotes a fraction of time $l(h)$ to work and the residual $1-l(1-h)$ to leisure. In aggregate terms, when $N$ denotes the total available labor force, the labor input of unskilled workers is $u N l$ and of skilled workers ( $1-u$ ) Nh. We divide the production function by $N$ and get output in per capita terms:

$$
y=k^{\alpha}\left[\left(A_{u} u l\right)^{\sigma}+\left(A_{s}(1-u) h\right)^{\sigma}\right]^{(1-\alpha) / \sigma} .
$$

First, we present the production function in a form which is convenient for the further analysis:

$$
y=A^{1-\alpha} k^{\alpha}\left[(\phi u l)^{\sigma}+((1-\phi)(1-u) h)^{\sigma}\right]^{(1-\alpha) / \sigma} .
$$

A denotes total factor productivity and $\phi$ and $1-\phi$ denotes the productivity of unskilled and skilled labor, ${ }^{4}$ respectively. After profit maximization, thus differentiation of Eq. (2.3) with respect to the inputs $u l,(1-u) h$ and $k$, we obtain the wages and interest rate in equilibrium:

$$
\begin{aligned}
& w_{l}=(1-\alpha) A^{1-\alpha} k^{\alpha}\left[(\phi u l)^{\sigma}+((1-\phi)(1-u) h)^{\sigma}\right]^{(1-\alpha) / \sigma-1} \phi^{\sigma}(u l)^{\sigma-1} \\
& w_{h}=(1-\alpha) A^{1-\alpha} k^{\alpha}\left[(\phi u l)^{\sigma}+((1-\phi)(1-u) h)^{\sigma}\right]^{(1-\alpha) / \sigma-1}(1-\phi)^{\sigma}((1-u) h)^{\sigma-1} \\
& r=\alpha A^{1-\alpha} k^{\alpha-1}\left[(\phi u l)^{\sigma}+((1-\phi)(1-u) h)^{\sigma}\right]^{(1-\alpha) / \sigma} .
\end{aligned}
$$

Besides the capital input the firms also need the labor input of skilled and unskilled workers. During the process of skillbiased technological change firms adopt technologies which make skilled workers more productive. This has an effect on the skill premium, which we obtain by dividing the skilled workers' wage $w_{h}$ by the unskilled workers' wage $w_{l}$ :

$$
\frac{w_{h}}{w_{l}}=\left(\frac{1-\phi}{\phi}\right)^{\sigma}\left(\frac{1-u}{u} \frac{h}{l}\right)^{\sigma-1} \text {. }
$$

As usual in this kind of models, the skill premium is increasing in $1-\phi$ and decreasing in $(1-u) h$.

\footnotetext{
4 Another way to think about $\phi$ is the "erosion effect" proposed by Galor and Moav (2000). New technology makes some of the old skills obsolete. Skilled workers are able to exploit technological change better than unskilled ones. Due to technological change the skilled workers become more productive, while unskilled workers lose productivity.
} 


\subsection{The households}

The household consists of skilled and unskilled workers and maximizes the total utility of its members. Utility stems from aggregate consumption and from leisure of both types of workers. We model the household side similar to Prasad (1996). Income is equally divided between skilled and unskilled household members. This is a strong simplification, as significant income differences between skilled and unskilled workers' households can be observed. Usually both kinds of workers live in separate households and only a small fraction of the skilled workers' income is transferred to the unskilled workers. However, the discussion on transfers between two types of households would add another decision variable, which would affect the decision on how much to work. We also neglect unemployment insurance and employment protection, which Mortensen and Pissarides (1999) use to explain how SBTC shocks lead to increased unemployment of unskilled workers. For the point that we make in this paper our simplified assumption is convenient and it is necessary in order to keep the model simple and tractable.

Workers can work only a fixed and given amount of hours, but they can choose the probability with which they supply labor. By law of large numbers we transform the probabilities into fractions of time devoted to work. As in the real business cycle literature, one week consists of $100 \mathrm{~h}$, which workers can supply to the production process (see for example Prescott, 2004). People tend to work around $40-50 \mathrm{~h}$ a week. When the average hours are multiplied by the probability to work and transformed into a fraction of the $100 \mathrm{~h}$ week, we get values for the labor input $h$ and $l$ which lie in the range $(0,1)$. In the model workers are endowed with one unit of time. As discussed in Section 2.1 the unskilled worker devotes a fraction of time $0<l<1$ to work and the residual $1-l$ to leisure, thus labor supply and leisure add up to 1 . Moreover, the skilled worker takes the same decision and devotes a fraction $0<h<1$ of his time to work and the residual time to leisure $1-h$. The constraint $l+(1-l)=1$ and $h+(1-h)=1$ always holds, thus workers use all available time. As described above, the household consists of a fraction $u$ of unskilled workers and a fraction $(1-u)$ of skilled workers. The individuals are born as skilled or unskilled workers. The considered time period is too short to study endogenous education choice and we assume that $u$ can change but is exogenously given. ${ }^{5}$ Each type of worker decides independently of the other type's decision, how much labor to supply. The household's utility function is given by

$$
U(c, 1-l, 1-h)=\ln c+B \psi u \ln (1-l)+B(1-\psi)(1-u) \ln (1-h) .
$$

The parameter $B$ is the weight on utility from leisure, $\psi$ determines how much weight is put on the utility of unskilled and skilled workers. We suppress the time index from now on. The size of the household grows with the rate $n$ and the subjective rate of time preferences is denoted by $\rho$. The household maximizes the discounted stream of utility: $\max _{c, l, h} \int_{t=0}^{\infty} \mathrm{e}^{-(\rho-n) t} U(c, 1-l, 1-h) \mathrm{d} t$ and has to obey the budget constraint:

$$
\dot{k}=w_{l} u l+w_{h}(1-u) h+r k-(\delta+n) k-c .
$$

We set up the Hamiltonian and solve it:

$$
\begin{aligned}
& H: \mathrm{e}^{-(\rho-n) t}\left[\ln c+B \psi u \ln (1-l)+B(1-\psi)(1-u) \ln \left(1-\frac{1}{c}=\lambda\right.\right. \\
& \frac{\partial H}{\partial c}=0 \Leftrightarrow \quad \mathrm{e}^{-(\rho-n) t} \frac{1}{c}=\lambda \quad \mathrm{e}^{-(\rho-n) t} \frac{B \psi u}{1-l}=\lambda w_{l} u \\
& \frac{\partial H}{\partial l}=0 \Leftrightarrow \quad \mathrm{e}^{-(\rho-n) t} \frac{B(1-\psi)(1-u)}{1-h}=\lambda w_{h}(1-u) \\
& \frac{\partial H}{\partial h}=0 \Leftrightarrow \quad r-\delta-n=-\frac{\dot{\lambda}}{\lambda} \\
& \frac{\partial H}{\partial k}=-\dot{\lambda} \Leftrightarrow \quad r \quad \lim _{t \rightarrow \infty} \mathrm{e}^{-(\rho-n) t} c^{-1} k=0 .
\end{aligned}
$$

After log-linearization of Eq. (2.11) and substitution in Eq. (2.14) we get the Euler equation

$$
\frac{\dot{c}}{c}=r-\delta-\rho .
$$

Now we combine Eqs. (2.11) and (2.12)

$$
\frac{B \psi}{1-l} \frac{1}{w_{l}}=\frac{1}{c}
$$

\footnotetext{
5 Based on data of the International Labor Organization (2008)http://laborsta.ilo.org, the annual average growth rate of the labor-force share with secondary and tertiary education was $0.5 \%$ and $0.43 \%$ points over the period $1996-2005$, for the Czech Republic, Hungary, Poland, the Slovak Republic and Slovenia. Also the US data show that the share of skilled labor force is increasing.
} 
and Eqs. (2.11) and (2.13)

$$
\frac{B(1-\psi)}{1-h} \frac{1}{w_{h}}=\frac{1}{c} .
$$

Moreover, we combine Eqs. (2.17) and (2.18),

$$
\frac{B \psi}{1-l} \frac{1}{w_{l}}=\frac{B(1-\psi)}{1-h} \frac{1}{w_{h}}
$$

which after rewriting leads to the optimal allocation of leisure.

$$
\frac{w_{h}}{w_{l}}=\frac{1-\psi}{\psi} \frac{1-l}{1-h} .
$$

From the firm sector we know the skill premium equation (2.7) and plug it into equation (2.20)

$$
\frac{1-\psi}{\psi} \frac{1-l}{1-h}=\left(\frac{1-\phi}{\phi}\right)^{\sigma}\left(\frac{1-u}{u} \frac{h}{l}\right)^{\sigma-1} .
$$

We rewrite it to obtain the optimal labor ratio for both kinds of workers

$$
\frac{1-\psi}{\psi}\left(\frac{1-\phi}{\phi}\right)^{-\sigma}\left(\frac{1-u}{u}\right)^{1-\sigma}=\frac{1-h}{1-l}\left(\frac{h}{l}\right)^{\sigma-1} .
$$

\subsubsection{Equilibrium}

In equilibrium firms maximize their profits, thus Eqs. (2.5)-(2.6) hold. The household maximizes its utility subject to the budget constraint and optimally allocates the available time of its skilled and unskilled members between labor supply and leisure. The resource constraint of the economy is given by the budget constraint and also by the fact that each type of worker spends all his time endowment on labor and leisure. In equilibrium the endogenous variables $c^{*}, k^{*}$ and $y^{*}$ grow at the same pace, and unless there is no shock to the skill-biased technology, the labor input of skilled and unskilled workers and their relative wages remain constant. We cannot provide an explicit solution of the equilibrium, because the state and control variables $k, c, h$ and $l$ mutually depend on each other. The numerical solution is presented in Section 4 . A comparative statics analysis of the equilibrium, namely a small increase of the skill-biased technology is performed in Appendix A.1. In the next step we introduce exogenous neutral technological change into the model.

\subsubsection{Equilibrium with SBTC and TFP growth}

In order to introduce neutral technological change into the model, we modify the production function. Total factor productivity is denoted as $A$ and its growth rate is $\dot{A} / A=g$. The current productivity depends on its initial level $A_{0}$ and the growth rate $g$, with $A=A_{0} \mathrm{e}^{g t}$. $A_{0}$ can be normalized to 1 , thus in any point in time $A=\mathrm{e}^{g t}$. Dividing the variables $y$, $k$ and $c$ by $A$ leads to output, capital and consumption per efficiency unit of labor. Now, output is called $\tilde{y}=y / A$, capital $\tilde{k}=k / A$ and consumption $\tilde{c}=c / A$. The production function becomes $\tilde{y}=\tilde{k}^{\alpha}\left[(\phi u l)^{\sigma}+((1-\phi)(1-u) h)^{\sigma}\right]^{(1-\alpha) / \sigma}$. After profit maximization, thus differentiation with respect to the inputs $u l,(1-u) h$ and $\tilde{k}$, we obtain:

$$
\begin{aligned}
& \tilde{w}_{l}=(1-\alpha) \tilde{k}^{\alpha}\left[(\phi u l)^{\sigma}+((1-\phi)(1-u) h)^{\sigma}\right]^{(1-\alpha) / \sigma-1} \phi^{\sigma}(u l)^{\sigma-1} \\
& \tilde{w}_{h}=(1-\alpha) \tilde{k}^{\alpha}\left[(\phi u l)^{\sigma}+((1-\phi)(1-u) h)^{\sigma}\right]^{(1-\alpha) / \sigma-1}(1-\phi)^{\sigma}((1-u) h)^{\sigma-1} \\
& \tilde{r}=\alpha \tilde{k}^{\alpha-1}\left[(\phi u l)^{\sigma}+((1-\phi)(1-u) h)^{\sigma}\right]^{(1-\alpha) / \sigma} .
\end{aligned}
$$

The budget constraint per efficiency units of labor becomes $y / A=c / A+\dot{k} / A+(n+\delta) k / A$. We use $\dot{\tilde{k}}=(k \dot{k})=\dot{k} / A-k \dot{A} / A^{2}$, plug it into the budget constraint and obtain after some rewriting

$$
\dot{\tilde{k}}=\tilde{w}_{l} u l+\tilde{w}_{h}(1-u) h+r \tilde{k}-(\delta+n+g) \tilde{k}-\tilde{c} .
$$

The control variable consumption is measured per efficiency units of labor, however the agents are interested in per capita consumption. This has to be considered in the utility function. Knowing that $c=\tilde{c} A=\tilde{c} \mathrm{e}^{g t}$, the objective function becomes: $\max _{\tilde{c}, l, h} \int_{t=0}^{\infty} \mathrm{e}^{-(\rho-n) t} U\left(\tilde{c} \mathrm{e}^{g t}, 1-l, 1-h\right) \mathrm{d} t$. We set up the Hamiltonian and solve it as described above.

$$
\begin{aligned}
H: & \mathrm{e}^{-(\rho-n) t}\left[\ln \left(\tilde{c} \mathrm{e}^{g t}\right)+B \psi u \ln (1-l)+B(1-\psi)(1-u) \ln (1-h)\right] \\
& +\lambda\left[\tilde{w}_{l} u l+\tilde{w}_{h}(1-u) h+r \tilde{k}-(\delta+n+g) \tilde{k}-\tilde{c}\right] .
\end{aligned}
$$


The resulting Euler equation is

$$
\frac{\dot{\tilde{c}}}{\tilde{\tilde{c}}}=r-\delta-\rho-g
$$

and as before,

$$
\frac{B \psi}{1-l} \frac{1}{\tilde{w}_{l}}=\frac{1}{\tilde{c}} \quad \text { and } \quad \frac{B(1-\psi)}{1-h} \frac{1}{\tilde{w}_{h}}=\frac{1}{\tilde{c}}
$$

describe the intra-temporal substitution between labor and consumption. Together with the TVC the unique optimal behavior in equilibrium is described. If we set $g=0$, the model is exactly as described in the previous section, since then $A=1$ in all periods. In order to analyze TFP growth, we set $g>0$. In order to obtain consumption and the capital stock in per capita terms, $\tilde{k}$ and $\tilde{c}$ have to be multiplied by $A$, which grows over time.

In equilibrium firms maximize profits, the household maximizes its utility and the budget constraint holds. If we assume skill-biased technological change away, under any plausible TFP growth rate the economy will stay on the balanced growth path and grow at the speed of exogenous technological progress. However, once skill-biased technology takes place, the economy moves to another equilibrium. The reader should be aware that no closed form solution exists, and only a numerical solution can be presented, which we do in Section 4.

\section{Empirics on SBTC}

In this section we calculate the time series for SBTC in Poland and the US. A simple method to obtain the productivity of unskilled workers $\phi$ from empirical data is to rewrite the skill premium equation (2.7):

$$
\frac{1-\phi}{\phi}=\left(\frac{w_{h}}{w_{l}}\right)^{1 / \sigma}\left(\frac{1-u}{u} \frac{h}{l}\right)^{(1-\sigma) / \sigma}
$$

Let us call the right hand side $D$, thus $(1-\phi) / \phi=D$. Solving for $\phi$ we get $\phi=\frac{1}{1+D}$, which is

$$
\phi=\frac{1}{1+\left(\frac{w_{h}}{w_{l}}\right)^{1 / \sigma}\left(\frac{1-u}{u} \frac{h}{l}\right)^{(1-\sigma) / \sigma}} .
$$

We observe the right hand side variables $u, h, l$ and the skill premium, but need to make an assumption on the elasticity of substitution. Following Caselli and Coleman (2006) we first set it to 1.4 , thus $\sigma=0.285$. The exercise is repeated with other values for the elasticity, ranging in the interval $(1,2)$ as proposed by Autor et al. (1998). The parameter $\sigma$ is exogenous, and once fixed it remains constant. One can argue that the elasticity of substitution depends on the share of skilled workers in total population. Because we do not have enough data to calibrate this parameter, we leave the elasticity constant.

\subsection{Empirics on SBTC in Poland}

Using empirical data for Poland ${ }^{6}$ in the period 1996-2006 we calculate the time series for $\phi$. Data comes from the Polish Central Statistical Office (2007). We focus only on full-time workers and look at the whole economy, because then the data on wages and the data on economically active people (from which we calculate $u$ ) can be easily connected. In total we have average wages for around 3-4 million workers. Workers are divided into five classes of education levels: tertiary (1), post-secondary (2), vocational secondary (3), general secondary (4) and primary (5).

We first analyze the private sector. The development of the productivity of unskilled workers is presented for different definitions of skilled workers. First, we consider workers with tertiary or post-secondary education as skilled and try different values of the elasticity of substitution. The ratio between skilled/unskilled employed workers, the skill premium and the skill-biased technology parameter $\phi$ is presented in Table 3.1.

There seems to be a rise in the proportion of skilled labor input to unskilled labor input as well as in the skill premium, which is accompanied by a decline in unskilled technology $\phi$. We also use other definitions of skilled workers. The left side of Table 3.2 presents results under the assumption that all workers but those with basic education (level 5) are skilled. The right side of the table shows another extreme, where we treat only those with tertiary education (level 1$)$ as skilled. The elasticity is set equal to 2 , thus $\sigma=0.5$.

Finally we analyze the whole labor market, including the private and public sector. Workers with general secondary education and below (levels 4,5 ) are considered as unskilled. Workers with at least vocational education (levels $1,2,3)$ are considered as skilled. Here $u$ is the share of unskilled workers in the total economically active population. The variables $h$ and $l$ are calculated as the number of full-time employed workers divided by the economically active population in a given 
Table 3.1

Development of skilled/unskilled labor supply, skill premium and $\phi$, private sector, Poland.

\begin{tabular}{|c|c|c|c|c|c|}
\hline \multirow[t]{2}{*}{ Year } & \multicolumn{2}{|l|}{$1,2-3,4,5$} & \multirow{2}{*}{$\begin{array}{l}\sigma=0.285 \\
\phi\end{array}$} & \multirow{2}{*}{$\begin{array}{l}\sigma=0.4 \\
\phi\end{array}$} & \multirow{2}{*}{$\begin{array}{l}\sigma=0.5 \\
\phi\end{array}$} \\
\hline & $(1-u) h / u l$ & $w_{h} / w_{l}$ & & & \\
\hline 1996 & 0.544 & 1.505 & 0.523 & 0.473 & 0.448 \\
\hline 1998 & 0.575 & 1.557 & 0.458 & 0.431 & 0.417 \\
\hline 1999 & 0.646 & 1.636 & 0.348 & 0.360 & 0.367 \\
\hline 2001 & 0.799 & 1.691 & 0.218 & 0.274 & 0.304 \\
\hline 2002 & 0.828 & 1.704 & 0.198 & 0.259 & 0.294 \\
\hline 2004 & 0.930 & 1.719 & 0.152 & 0.224 & 0.267 \\
\hline 2006 & 0.997 & 1.905 & 0.095 & 0.167 & 0.217 \\
\hline
\end{tabular}

Table 3.2

Development of skilled/unskilled labor supply, skill premium and $\phi$ under $\sigma=0.5$, for two different definitions of skilled/unskilled workers, private sector, Poland.

\begin{tabular}{|c|c|c|c|c|c|c|}
\hline \multirow[t]{2}{*}{ Year } & \multicolumn{3}{|l|}{$1,2,3,4-5$} & \multicolumn{3}{|l|}{$1-2,3,4,5$} \\
\hline & $(1-u) h / u l$ & $w_{h} / w_{l}$ & $\phi$ & $(1-u) h / u l$ & $w_{h} / w_{l}$ & $\phi$ \\
\hline 1996 & 4.752 & 1.278 & 0.114 & 0.092 & 2.215 & 0.690 \\
\hline 1998 & 5.335 & 1.315 & 0.098 & 0.098 & 2.397 & 0.639 \\
\hline 1999 & 5.886 & 1.398 & 0.080 & 0.113 & 2.486 & 0.588 \\
\hline 2001 & 7.319 & 1.482 & 0.059 & 0.155 & 2.434 & 0.521 \\
\hline 2002 & 8.133 & 1.505 & 0.052 & 0.166 & 2.441 & 0.503 \\
\hline 2004 & 10.593 & 1.473 & 0.042 & 0.205 & 2.333 & 0.473 \\
\hline 2006 & 11.733 & 1.400 & 0.042 & 0.237 & 2.413 & 0.420 \\
\hline
\end{tabular}

Table 3.3

Development of skilled/unskilled labor supply, wage and $\phi$ under $\sigma=0.4$ and 0.5 , whole economy, Poland.

\begin{tabular}{|c|c|c|c|c|c|}
\hline \multirow[t]{2}{*}{ Year } & \multicolumn{3}{|l|}{$1,2,3-4,5$} & \multirow{2}{*}{$\begin{array}{l}\sigma=0.4 \\
\phi\end{array}$} & \multirow{2}{*}{$\begin{array}{l}\sigma=0.5 \\
\phi\end{array}$} \\
\hline & $(1-u) h / u l$ & $w=w_{h} / w_{l}$ & $u$ & & \\
\hline 1996 & 1.086 & 1.288 & 0.559 & 0.319 & 0.357 \\
\hline 1998 & 1.242 & 1.380 & 0.537 & 0.244 & 0.297 \\
\hline 1999 & 1.339 & 1.422 & 0.503 & 0.211 & 0.270 \\
\hline 2001 & 1.586 & 1.479 & 0.509 & 0.158 & 0.224 \\
\hline 2002 & 1.622 & 1.518 & 0.497 & 0.146 & 0.211 \\
\hline 2004 & 1.860 & 1.614 & 0.462 & 0.106 & 0.171 \\
\hline 2006 & 1.982 & 1.564 & 0.419 & 0.105 & 0.171 \\
\hline
\end{tabular}

skill category. The results under different elasticities of substitution between skilled and unskilled workers are presented in Table 3.3.

Empirical data confirms the presence of skill-biased technological change in Poland. We apply the same approach to the most developed economy, namely the US. This allows us to state that the observation for Poland is in line with the general development in advanced economies and is not an exception.

\subsection{Empirics on SBTC in the US}

We calculate the productivity of unskilled workers for the US over the period 1975-2008. The data ${ }^{7}$ comes from the US Bureau of Labour Statistics (2008). The education attainment levels are (1) college graduates, (2) less than a bachelor's degree, (3) high school graduates, no college and (4) less than a high school diploma. Wages are the median wages for the whole population. As before we set the elasticity of substitution equal to 2 and calculate the skill-biased technology parameter $\phi$ with Eq. (3.2). Workers with high school diploma or less are considered as unskilled. The data on SBTC shows the following pattern. In 1975 the productivity of unskilled workers $\phi$ was 0.45 . It declined in a nearly linear way until 1993 and took the value 0.22 from then. From there on SBTC can still be observed, however the change of $\phi$ is much slower. The productivity of unskilled workers $\phi$ takes the value of 0.21 in 2000 and declines to 0.18 in 2008 .

Data availability allows us to look at the share of skilled workers in the labor force in the period 1992-2008. The share of unskilled workers $u$ was 0.48 in 1992, 0.42 in 2000 and decreases continuously, reaching 0.38 in 2008 . As in Poland, an upgrading of skills can be observed. It is important to explain why workers decide to invest in education, but this is beyond the scope of the paper.

\footnotetext{
6 We obtain the longest time series among the Central and Eastern European countries for Poland from the Polish Central Statistical Office (2007). For the other CEE countries data is present since 2004 only.

7 Recent data can be found on http://www.bls.gov/cps/earnings.htm\#education, older data need to be requested by email.
} 


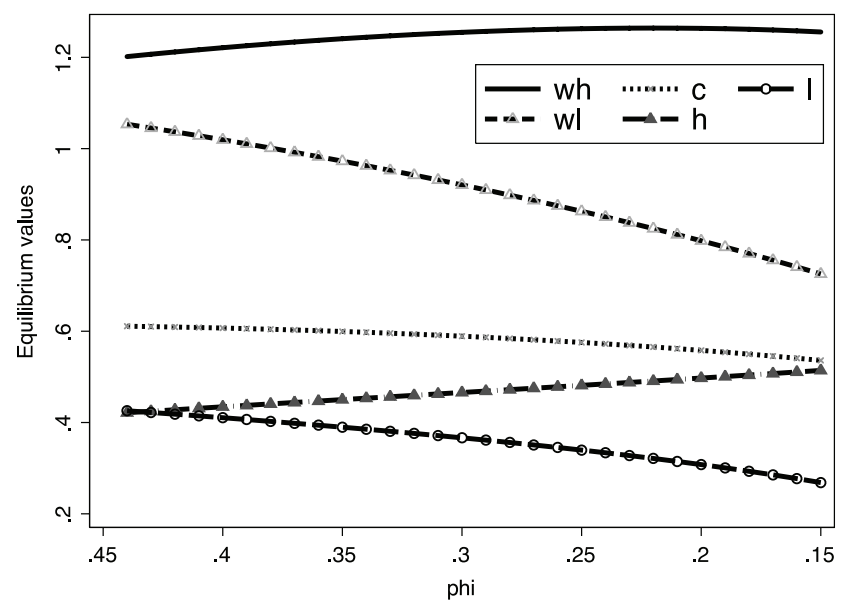

Fig. 4.1. Equilibrium values for $w_{h}, w_{l}, c, h$ and $l$ (top to bottom) under skill-biased technological change, thus for decreasing $\phi$ values, Poland.

\section{Model calibration and numerical analysis of the equilibrium}

We calibrate the model based on empirical data and test whether it is consistent with empirical observations. First, we calculate the unobserved skill-biased technology parameter $\phi$ using the previously presented method and calibrate the model. In a second step, we perform a comparative statics analysis, namely fix the TFP growth rate and study the equilibrium under different $\phi$ values. The economy in equilibrium is described by seven equations with seven unknown variables. The variables of interest are: $k, c, h, l, w_{h}, w_{l}$ and $r$. The seven equations are the optimality conditions in the firm sector (Eqs. (2.4)-(2.6)), the budget constraint (2.9), the Euler equation (2.16), and finally the substitution of marginal utility from leisure and consumption ((2.17) and (2.18)).

We have to calibrate some parameters and we apply the following procedure. The capital share is set to $\alpha=0.35$. The elasticity of substitution is set to $2(\sigma=0.5)$. The initial TFP level $A$ is arbitrarily set to 1 , it only scales up $k, c, w_{h}$ and $w_{l}$, but has no effect on $h$ and $l$. Moreover, the share of unskilled workers $u$ is set to 0.509, a value observed in 2001 in Poland. We still need to calibrate the parameters $B, \psi, \delta$ and $\rho$. The rate of time preferences $\rho$ is set equal to 0.065 (see Benhabib and Perli (1994) and Benhabib and Farmer (1994)) and the depreciation of physical capital $\delta$ is set equal to 0.06 (see King and Rebelo (1999) and Stokey and Rebelo (1995)).

\subsection{Calibration for Poland}

First, we apply empirical data for Poland to Eq. (2.20) and get $\psi$. The relative weight of the utility of unskilled workers in total utility of the household is calibrated as $\psi=0.46$. This value is in line with Prasad's specification, who sets it equal to 0.4. The parameter $B$ is set to match Eqs. (2.17) and (2.18) with empirical data and the procedure is as follows. In equilibrium $\dot{c}=0$ holds, thus $r=\delta+\rho$ holds. We apply this fact and the observed labor input $h$ and $l$ for different $\phi$ values from Table 3.3 to the interest rate equation (2.6) and find $k^{*}$. Now we plug $k^{*}$ into $\dot{k}=0$ to find $c^{*}$. Applying $c^{*}$ to Eqs. (2.17) and (2.18) we calibrate the weight on utility from leisure as $B=2.12783$. Finally, we use the just calibrated parameters to find the wages $w_{l}$ and $w_{h}$.

Using the calibrated parameters $\sigma, \psi, B, \alpha, \delta$ and $\rho$, which are kept fixed, the seven equations with seven unknowns are solved numerically. This procedure is repeated for $\phi$ in the range $0.15-0.45$, where $\phi$ decreases by very small values. We obtain a monotone function for the variables $k, h, l, c$ and $w_{l}$ and plot it. Only the skilled workers' wage $w_{h}$ shows a maximum at $\phi=0,22$. The exercise is performed with steps of size 0.0001 . Table 4.1 presents the results using steps of 0.01 . The variables in equilibrium are presented in Fig. 4.1, the variable $k$ is omitted to improve legibility.

In equilibrium, the variables behave as predicted ${ }^{8}$ by the comparative statics analysis on $\mathrm{d} l / \mathrm{d} \phi, \mathrm{d} h / \mathrm{d} \phi$ and $\mathrm{d} k / \mathrm{d} \phi$ in Appendix A.1. Increasing the productivity of the skilled workers and decreasing that of the unskilled workers results in a higher skilled labor input $h$ and lower unskilled labor input $l$ as well as in an increased skill premium. We also observe a decline in physical capital and consumption. This is only due to the fact that TFP is kept constant. Under our setting of parameters SBTC decreases the productivity of unskilled workers more than it increases that of skilled workers, in relative terms (e.g. a change from $\phi=0.2$ and $1-\phi=0.8$ by 0.01 means a decrease by $5 \%$ and an increase by $1.25 \%$ ). We keep $u=0.509$ constant, the population consists of slightly more unskilled workers than skilled workers. Consequently, under the above setting, SBTC decreases the output in equilibrium if it is not accompanied by TFP growth.

We now study the case of SBTC and TFP growth, thus $\phi$ decreases and $A$ increases. Explicitly, we connect a 0.01 decline in $\phi$ with an increase of $A$ by $2 \%$, starting from $\phi=0.44$ and $A=1$. This is a simplification, which makes the analysis

\footnotetext{
8 Comparing the results of the two different approaches, we have to bear in mind that in the above analysis the ratio between $h$ and $l$ changes over time. However, it is fixed for simplicity when we perform the comparative statics analysis in Appendix A.1.
} 
Table 4.1

Values of $k, h, l, w_{h}, w_{l}$ and $c$, in equilibrium, under fixed $A=1$, Poland.

\begin{tabular}{|c|c|c|c|c|c|c|}
\hline$\phi$ & $k$ & $h$ & $l$ & $w_{h}$ & $w_{l}$ & $c$ \\
\hline 0.44 & 2.0562 & 0.421487 & 0.425786 & 1.20168 & 1.0534 & 0.610986 \\
\hline 0.43 & 2.05336 & 0.42476 & 0.42208 & 1.20685 & 1.0452 & 0.610142 \\
\hline 0.42 & 2.05017 & 0.428009 & 0.418311 & 1.21182 & 1.03681 & 0.609193 \\
\hline 0.41 & 2.04662 & 0.431236 & 0.414474 & 1.21658 & 1.02823 & 0.608137 \\
\hline 0.4 & 2.0427 & 0.434444 & 0.410566 & 1.22114 & 1.01946 & 0.606974 \\
\hline 0.39 & 2.03842 & 0.437634 & 0.406583 & 1.22549 & 1.0105 & 0.605701 \\
\hline 0.38 & 2.03377 & 0.440808 & 0.40252 & 1.22963 & 1.00133 & 0.604319 \\
\hline 0.36 & 2.02333 & 0.447114 & 0.394135 & 1.23728 & 0.98241 & 0.601219 \\
\hline 0.35 & 2.01754 & 0.450251 & 0.389802 & 1.24078 & 0.972642 & 0.599498 \\
\hline 0.34 & 2.01136 & 0.45338 & 0.385369 & 1.24405 & 0.962666 & 0.597661 \\
\hline 0.33 & 2.00477 & 0.456501 & 0.380828 & 1.2471 & 0.952479 & 0.595704 \\
\hline 0.32 & 1.99778 & 0.459619 & 0.376173 & 1.24992 & 0.942074 & 0.593627 \\
\hline 0.31 & 1.99038 & 0.462733 & 0.371397 & 1.25251 & 0.93145 & 0.591426 \\
\hline 0.3 & 1.98254 & 0.465847 & 0.36649 & 1.25485 & 0.920599 & 0.589099 \\
\hline 0.29 & 1.97428 & 0.468962 & 0.361446 & 1.25695 & 0.909518 & 0.586642 \\
\hline 0.27 & 1.95639 & 0.475205 & 0.350902 & 1.26038 & 0.88664 & 0.581329 \\
\hline 0.26 & 1.94675 & 0.478338 & 0.345381 & 1.2617 & 0.874829 & 0.578464 \\
\hline 0.25 & 1.93663 & 0.481482 & 0.339676 & 1.26275 & 0.862761 & 0.575455 \\
\hline 0.24 & 1.926 & 0.484638 & 0.333775 & 1.26351 & 0.850426 & 0.572297 \\
\hline 0.23 & 1.91485 & 0.487811 & 0.327662 & 1.26398 & 0.837816 & 0.568985 \\
\hline 0.22 & 1.90317 & 0.491003 & 0.321318 & 1.26415 & 0.824921 & 0.565513 \\
\hline 0.21 & 1.89092 & 0.494217 & 0.314726 & 1.264 & 0.811729 & 0.561875 \\
\hline 0.2 & 1.8781 & 0.497456 & 0.307861 & 1.26352 & 0.798228 & 0.558064 \\
\hline 0.19 & 1.86467 & 0.500725 & 0.300701 & 1.26269 & 0.784404 & 0.554072 \\
\hline 0.18 & 1.8506 & 0.504027 & 0.293218 & 1.26151 & 0.770242 & 0.549891 \\
\hline 0.17 & 1.83586 & 0.507367 & 0.285378 & 1.25994 & 0.755725 & 0.545512 \\
\hline 0.16 & 1.82041 & 0.510749 & 0.277146 & 1.25798 & 0.740833 & 0.540923 \\
\hline 0.15 & 1.80422 & 0.51418 & 0.26848 & 1.2556 & 0.725546 & 0.536112 \\
\hline
\end{tabular}

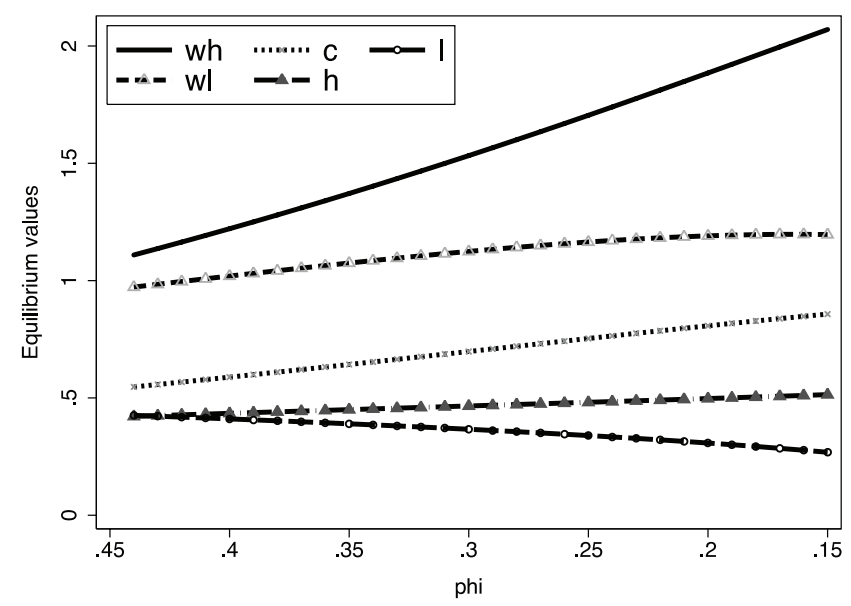

Fig. 4.2. Equilibrium values for $w_{h}, w_{l}, c, h$ and $l$ (top to bottom) under skill-biased technological change, thus for decreasing $\phi$ values, under TFP growth, Poland.

significantly easier and the results more transparent. We know from empirical studies that exogenous TFP growth can be set to around $2 \%$ per year (see e.g. Burda and Severgnini (2009)). The parameter B needs to be calibrated again. Now it is calibrated as $B=2.19403$. This value is slightly higher than in the case where TFP does not grow. As workers become more productive, they need to spend less time in the production process, in order to keep the output constant. If they are aware of the constant productivity growth, they value leisure a little bit more than they would if no technological progress were present.

We see that under this setting a decrease of $\phi$ leads to a higher employment of the skilled workers, lower employment of the unskilled workers, a higher capital stock and consumption. This result is presented in Table 4.2 and shown in Fig. 4.2 . We find that consumption and the capital stock grow, if SBTC is accompanied by a sufficiently high TFP growth.

Finally, we test the empirical relevance of the model. Fixing $A=1$ and using the same parameters as described above, we calculate the equilibrium for all observed years. We apply the productivity $\phi$ of unskilled workers and the share $u$ of unskilled workers in the economic active population that is actually observed in the given year. We calculate $\phi$ with 
Table 4.2

Values of $k, h, l, w_{h}, w_{l}$ and $c$, in equilibrium, under increasing $A$, Poland.

\begin{tabular}{|c|c|c|c|c|c|c|}
\hline$\phi$ & $k$ & $h$ & $l$ & $w_{h}$ & $w_{l}$ & $c$ \\
\hline 0.44 & 1.63643 & 0.421483 & 0.425787 & 1.10938 & 0.972488 & 0.547036 \\
\hline 0.43 & 1.66718 & 0.424756 & 0.422081 & 1.13667 & 0.984408 & 0.557315 \\
\hline 0.42 & 1.69822 & 0.428005 & 0.418312 & 1.1644 & 0.996235 & 0.567689 \\
\hline 0.41 & 1.72952 & 0.431232 & 0.414475 & 1.19259 & 1.007955 & 0.578156 \\
\hline 0.4 & 1.76108 & 0.43444 & 0.410567 & 1.22125 & 1.019544 & 0.588706 \\
\hline 0.39 & 1.7929 & 0.43763 & 0.406584 & 1.25036 & 1.030989 & 0.599338 \\
\hline 0.38 & 1.82495 & 0.440803 & 0.40252 & 1.27993 & 1.042284 & 0.610053 \\
\hline 0.37 & 1.8572 & 0.443963 & 0.398373 & 1.30995 & 1.053392 & 0.620835 \\
\hline 0.36 & 1.88967 & 0.44711 & 0.394135 & 1.34044 & 1.064315 & 0.63169 \\
\hline 0.35 & 1.92233 & 0.450247 & 0.389803 & 1.37139 & 1.075022 & 0.642608 \\
\hline 0.34 & 1.95514 & 0.453376 & 0.38537 & 1.40278 & 1.085486 & 0.653577 \\
\hline 0.33 & 1.98812 & 0.456497 & 0.380829 & 1.43464 & 1.095699 & 0.664601 \\
\hline 0.32 & 2.02121 & 0.459614 & 0.376174 & 1.46692 & 1.105621 & 0.67566 \\
\hline 0.31 & 2.05439 & 0.462729 & 0.371397 & 1.49965 & 1.115235 & 0.686754 \\
\hline 0.3 & 2.08765 & 0.465843 & 0.366491 & 1.53281 & 1.124511 & 0.69787 \\
\hline 0.29 & 2.12094 & 0.468958 & 0.361447 & 1.56639 & 1.133419 & 0.709 \\
\hline 0.28 & 2.15424 & 0.472077 & 0.356254 & 1.60038 & 1.141929 & 0.720132 \\
\hline 0.27 & 2.18751 & 0.475201 & 0.350903 & 1.63477 & 1.150002 & 0.731251 \\
\hline 0.26 & 2.2207 & 0.478334 & 0.345381 & 1.66954 & 1.157603 & 0.742346 \\
\hline 0.25 & 2.25377 & 0.481478 & 0.339677 & 1.70467 & 1.164691 & 0.7534 \\
\hline 0.24 & 2.28668 & 0.484634 & 0.333776 & 1.74016 & 1.171232 & 0.764403 \\
\hline 0.23 & 2.31938 & 0.487807 & 0.327663 & 1.77598 & 1.177178 & 0.775332 \\
\hline 0.22 & 2.3518 & 0.490999 & 0.321319 & 1.81209 & 1.182477 & 0.786171 \\
\hline 0.21 & 2.38385 & 0.494213 & 0.314726 & 1.84847 & 1.187067 & 0.79689 \\
\hline 0.2 & 2.41552 & 0.497452 & 0.307862 & 1.8851 & 1.190905 & 0.807474 \\
\hline 0.19 & 2.4467 & 0.500721 & 0.300702 & 1.92193 & 1.193924 & 0.817895 \\
\hline 0.18 & 2.47729 & 0.504023 & 0.293218 & 1.95891 & 1.196055 & 0.828122 \\
\hline 0.17 & 2.50721 & 0.507363 & 0.285379 & 1.99601 & 1.197219 & 0.838123 \\
\hline 0.16 & 2.53634 & 0.510746 & 0.277147 & 2.03316 & 1.197334 & 0.847858 \\
\hline 0.15 & 2.56456 & 0.514176 & 0.268481 & 2.07031 & 1.196316 & 0.857296 \\
\hline
\end{tabular}

Table 4.3

Simulated and observed development of skilled/unskilled labor supply and skill premium under $\sigma=0.5$, whole economy, Poland.

\begin{tabular}{|c|c|c|c|c|c|c|}
\hline \multirow[t]{2}{*}{ Year } & \multicolumn{3}{|c|}{ Observed $1,2,3-4,5$} & \multirow[b]{2}{*}{$\phi$} & \multicolumn{2}{|l|}{ Simulated } \\
\hline & $(1-u) h / u l$ & $w=w_{h} / w_{l}$ & $u$ & & $(1-u) h / u l$ & $\begin{array}{l}w= \\
w_{h} / w_{l}\end{array}$ \\
\hline 1996 & 1.086 & 1.288 & 0.559 & 0.357 & 0.982 & 1.354 \\
\hline 1998 & 1.242 & 1.380 & 0.537 & 0.297 & 1.168 & 1.424 \\
\hline 1999 & 1.339 & 1.422 & 0.503 & 0.270 & 1.338 & 1.421 \\
\hline 2001 & 1.586 & 1.479 & 0.509 & 0.224 & 1.473 & 1.534 \\
\hline 2002 & 1.622 & 1.518 & 0.497 & 0.211 & 1.569 & 1.544 \\
\hline 2004 & 1.860 & 1.614 & 0.462 & 0.171 & 1.927 & 1.586 \\
\hline 2006 & 1.982 & 1.564 & 0.419 & 0.171 & 2.127 & 1.510 \\
\hline
\end{tabular}

Eq. (3.2) and data from the Polish Central Statistical Office (2007). Using this value, we calculate the values of $k, h, l, w_{h}, w_{l}$ and $c$ in equilibrium. We compare the calculated ratio of skilled to unskilled labor input $(1-u) h / u l$ and the skill premium $w_{h} / w_{l}$ with its empirical value. The results are presented in Table 4.3. The calculated values follow the empirical values quite well. Differences can be observed, but they origin from the assumption of perfect competitive equilibrium. The model simulates the agents' optimal behavior in equilibrium, while the empirical data is just very close to the equilibrium. Employment laws, government interventions and other economic factors which are not captured in the model make the agents choose allocations that differ from our equilibrium. Moreover, our calibration is based on very few observations. Further on, we do not observe the real hours worked, but calculate $h$ as the share of full-time, skilled workers among all economically active skilled workers. The same applies to $l$.

The numerical analysis shows that the model is useful in explaining that SBTC and TFP growth lead to output growth and increasing employment of skilled workers and declining employment of unskilled workers.

\subsection{Calibration for the US}

Calibrating the model to the US shows that it is also empirically relevant for the most advanced economy. The calibration is performed as in the case of Poland. Data on wages, employment and unemployment origins from the US Bureau of Labour Statistics (2008) and covers the period 1992-2008. The elasticity of substitution is set equal to $2(\sigma=0.5)$ and 
Table 4.4

Simulated and observed development of skilled/unskilled labor supply, and skill premium under $\sigma=0.5$, US.

\begin{tabular}{|c|c|c|c|c|c|c|c|c|}
\hline \multirow[t]{2}{*}{ Year } & \multicolumn{3}{|l|}{ Observed } & \multirow[b]{2}{*}{$\phi$} & \multicolumn{2}{|c|}{ Simulated on $1992-1998$} & \multicolumn{2}{|c|}{$\begin{array}{l}\text { Simulated on full } \\
\text { sample }\end{array}$} \\
\hline & $(1-u) h / u l$ & $w_{h} / w_{l}$ & $u$ & & $(1-u) h / u l$ & $w_{h} / w_{l}$ & $(1-u) h / u l$ & $\overline{w_{h} / w_{l}}$ \\
\hline 2000 & 1.419 & 1.598 & 0.418 & 0.216 & 1.437 & 1.588 & 1.437 & 1.588 \\
\hline 2001 & 1.447 & 1.600 & 0.414 & 0.212 & 1.477 & 1.598 & 1.465 & 1.598 \\
\hline 2002 & 1.469 & 1.598 & 0.411 & 0.210 & 1.507 & 1.578 & 1.484 & 1.590 \\
\hline 2003 & 1.498 & 1.582 & 0.406 & 0.211 & 1.499 & 1.581 & 1.499 & 1.581 \\
\hline 2004 & 1.526 & 1.573 & 0.402 & 0.209 & 1.520 & 1.577 & 1.520 & 1.577 \\
\hline 2005 & 1.535 & 1.585 & 0.400 & 0.206 & 1.541 & 1.584 & 1.541 & 1.584 \\
\hline 2006 & 1.560 & 1.599 & 0.396 & 0.201 & 1.603 & 1.577 & 1.578 & 1.589 \\
\hline 2007 & 1.629 & 1.622 & 0.385 & 0.189 & 1.672 & 1.601 & 1.672 & 1.601 \\
\hline 2008 & 1.673 & 1.628 & 0.380 & 0.184 & 1.744 & 1.594 & 1.717 & 1.606 \\
\hline
\end{tabular}

the parameters that enter the utility function are calibrated as $\psi=0.3912$ and $B=2.4375$ to match empirical data. Those parameters are similar to those obtained for Poland. The weight of the utility of skilled workers in the households' total utility seems to be higher in the US. However, this can be affected by the definition of skilled workers. The workers in the US seem to put a higher weight $B$ on the utility from leisure compared to that from consumption than workers in Poland do. In advanced economies leisure becomes more valuable than consumption, possibly because of the very high consumption level and scarcity of leisure. The US data shows a very high employment rate.

We test the empirical relevance of the model. First, we calibrate the parameters based on the whole sample. Using the calibrated parameters and observed skill-biased technology parameter $\phi$ we calculate the skill premium and ratio of skilled to unskilled labor supply for the period 1992-2008. The observed and simulated values are presented in Table 4.4. The simulated skill premium is $0.5 \%$ lower while the labor supply of skilled workers relative to unskilled ones is $1.5 \%$ higher, on average. During the second test we calibrate the parameters for the period 1992-1998 and predict the development of the economy in the following period. In this case, the parameters are calibrated as $\psi=0.39518$ and $B=2.4681$. The average share of unskilled workers in the labor force for the first period is $u=0.449$ and $u=0.401$ for the second. Now, the simulated skill premium is $1.5 \%$ lower than the observed one, on average. The labor supply of skilled to unskilled workers is by $3 \%$ higher. Considering the relatively small error, the model is useful in predicting the reaction of the labor market to a skill-biased technological change. Policy makers can predict the shares of skilled workers quite well, as they observe the enrollment in schools and universities. Once the model is calibrated for a country, it can be used to predict the reaction of unskilled workers to SBTC. Policy makers can take appropriate actions in order to sustain the welfare of unskilled workers.

When we consider SBTC as an exogenous process the model can explain the formation of the skill premium. The labor supply of skilled and unskilled workers in the US is very similar and stays constant over time. However, the share of unskilled workers decreases over time, from 0.48 in 1992 to 0.38 in 2008. This indicates that it is crucial to include the share of unskilled workers in the population into the model. There is too few data to estimate the relation between SBTC and education attainment, however the data indicates that also in the US the increasing skill premium makes education attractive.

\section{Conclusion}

This paper relates the change in the structure of the labor market to skill-biased technological change and endogenous labor supply. Using elastic labor supply we show that skill-biased technological change can explain the observed increase of unemployment of unskilled workers. Further on, we calculate the time series of skill-biased technology for Poland and the US. Calibration of the model to Polish and US data and numerical analysis of the equilibrium shows its empirical relevance. The model explains the significant employment drop of unskilled workers in Poland.

We add to the results of the mainstream SBTC literature by making labor supply endogenous. We deal with a heterogeneous-agents model of economic growth and present numerical results. The major contribution of our model is the observation that if skill-biased technological change is not followed by total factor productivity growth, then output, physical capital stock and consumption decline. The model allows to simulate the economy and study the effect of SBTC on the supply and demand side of the labor market.

\section{Acknowledgments}

We thank Stefano Bosi and Jakub Growiec for some important remarks. Participants of the Warsaw International Economic Meeting 2008, Milan DEAS Workshop on Growth 2008, Bielefeld EBIM workshop 2008, the WISE seminar at University of Venice and the research seminar at Warsaw School of Economics made useful comments. 


\section{Appendix}

\section{A.1. Comparative statics - the impact of $\phi$ on $l$, $h$ and $k$}

In order to study the effect of a change in skill-biased technology parameter $\phi$ on unskilled and skilled labor demand and the physical capital stock, we take the total differential of the three firms' optimality conditions (2.4)-(2.6). This approach follows Cieślik (2008). We know from the households' optimality equation (2.20) that the supply of skilled and unskilled labor depends on the skill premium. After substituting the wage for skilled and unskilled labor and rewriting, we get Eq. (2.22), which we call $S$ :

$$
S=\frac{1-\psi}{\psi}\left(\frac{1-\phi}{\phi}\right)^{-\sigma}\left(\frac{1-u}{u}\right)^{1-\sigma}-\frac{1-h}{1-l}\left(\frac{h}{l}\right)^{\sigma-1}
$$

We use the following three equations which completely describe the economy: $S, w_{h}$ and $r$ and differentiate them with respect to $\phi, l, h$ and $k$. To simplify the notation, we use $w_{l}, w_{h}$ and $r$, and the second subscript denotes the first derivative with respect to the given parameter. In matrix notation we obtain:

$$
\left(\begin{array}{ccc}
S_{k} & S_{l} & S_{h} \\
w_{h k} & w_{h l} & w_{h h} \\
r_{k} & r_{l} & r_{h}
\end{array}\right)\left(\begin{array}{c}
\mathrm{d} k \\
\mathrm{~d} l \\
\mathrm{~d} h
\end{array}\right)=\left(\begin{array}{c}
-S_{\phi} \\
-w_{h \phi} \\
-r_{\phi}
\end{array}\right) \mathrm{d} \phi
$$

We solve this system of three linear equations for $\mathrm{d} k, \mathrm{~d} l$ and $\mathrm{d} h$, use the fact that $S_{k}=0$ and after rearranging the terms obtain the following expressions:

$$
\begin{aligned}
\frac{\mathrm{d} l}{\mathrm{~d} \phi} & =\frac{-w_{h k} S_{h} r_{\phi}+w_{h k} S_{\phi} r_{h}+S_{h} r_{k} w_{h \phi}-S_{\phi} r_{k} w_{h h}}{-w_{h k} S_{l} r_{h}+r_{l} w_{h k} S_{h}-w_{h l} r_{k} S_{h}+r_{k} S_{l} w_{h h}} \\
\frac{\mathrm{d} h}{\mathrm{~d} \phi} & =-\frac{r_{l} w_{h k} S_{\phi}-w_{h l} r_{k} S_{\phi}-w_{h k} S_{l} r_{\phi}+r_{k} S_{l} w_{h \phi}}{-w_{h k} S_{l} r_{h}+r_{l} w_{h k} S_{h}-w_{h l} r_{k} S_{h}+r_{k} S_{l} w_{h h}} \\
\frac{\mathrm{d} k}{\mathrm{~d} \phi} & =-\frac{w_{h h} S_{l} r_{\phi}-w_{h \phi} S_{l} r_{h}+S_{h} r_{l} w_{h \phi}-S_{h} w_{h l} r_{\phi}+S_{\phi} w_{h l} r_{h}-S_{\phi} r_{l} w_{h h}}{-w_{h k} S_{l} r_{h}+r_{l} w_{h k} S_{h}-w_{h l} r_{k} S_{h}+r_{k} S_{l} w_{h h}} .
\end{aligned}
$$

In order to study the impact of the skill-biased technological change, and hence the decrease of $\phi$, we need to determine the sign of equations Eqs. (A.2)-(A.4). It is easy to check that $S_{l}<0, S_{h}>0, S_{\phi}>0, w_{h k}>0, w_{h l}>0, w_{h h}<0, w_{h \phi}<0$, $r_{k}<0, r_{l}>0$ and $r_{h}>0, r_{\phi}>0$ holds. There are eight cases for the sign of Eqs. (A.2)-(A.4). $(+,+,+),(+,+,-),(+$, $-,-),(-,+,+),(-,+,-),(+,-,+),(-,-,+),(-,-,-)$. From the empirical point of view the case $(+,-,-)$ and $(+$, $-,+)$ should be studied. Under this setting an decrease in $\phi$ leads to a decline in unskilled labor, which is consistent with empirical observations.

The terms in Eqs. (A.2)-(A.4) are quite complicated and make an analytical solution infeasible. The partial derivatives are presented in Appendix A.2. Some of the parameters can be fixed: $\alpha=0.35, \psi=0.46, A=1, k=100$ and $u=0.5$. We explain the choice of the parameters in detail. The capital share is assumed to be constant and around 0.35 . The parameter $\psi$ is set to 0.46 in order to match Eq. (2.20) with empirical data for Poland. The TFP is set arbitrarily to 1, in order to simplify the equation. Also the capital stock $k$ is chosen arbitrarily, because this value does not change the results of the analysis $(A$ and $k$ disappear after simplification of Eqs. (A.2) and (A.3)). Finally, we treat workers with primary and secondary education as unskilled, while the remaining ones are skilled. The share of unskilled workers in the total population is quite close to 0.5 (see Table 3.3). We are left with $h, l$ and $\phi$. Based on empirical observations, we put the parameters in intervals: $0.2<l<0.5$, $0.3<h<0.5$ and $0.15<\phi<0.4$. The motivation is provided in Section 2.2 and empirical values for $\phi$ can be found in Table 3.3. Skilled workers work more than unskilled, thus we assume $l<h$. Due to data availability, we pretend that only full-time employed workers are employed and the rest is unemployed. The interesting point is to find the relation between $\phi, h$ and $l$, under which $\frac{\mathrm{d} l}{\mathrm{~d} \phi}>0$ and simultaneously $\frac{\mathrm{d} h}{\mathrm{~d} \phi}<0$. As no analytical closed form solution exists, we resort to graphical analysis. From empirical data for Poland we obtain an average relationship between $l$ and $h$. Thus, only two variables, namely $h$ and $\phi$ are left. A 3D graph of $\frac{\mathrm{d} l}{\mathrm{~d} \phi}$ and $\frac{\mathrm{d} h}{\mathrm{~d} \phi}$ is presented in Fig. A.1.

Repeating the procedure with slightly different parameter values results in qualitatively the same picture. For any value of $h$ there exists a range of the skill-biased technology $\phi$, such that $\frac{\mathrm{d} l}{\mathrm{~d} \phi}>0$ and simultaneously $\frac{\mathrm{d} h}{\mathrm{~d} \phi}<0$ holds. This range is the intersection of the two plots with the flat hyperplane that goes through zero (front of Fig. A.1). When $u$ decreases, this range moves to the left. When the ratio $l / h$ decreases, the intersection of $\frac{\mathrm{d} h}{\mathrm{~d} \phi}$ with the flat hyperplane that goes through zero moves to the right. Skill-biased technological change increases skilled labor supply and decreases unskilled labor supply. The function $\mathrm{d} k / \mathrm{d} \phi$ is not plotted. It is positive for any observable $\phi$ value. This means that SBTC decreases the equilibrium physical capital stock when TFP is kept constant. 


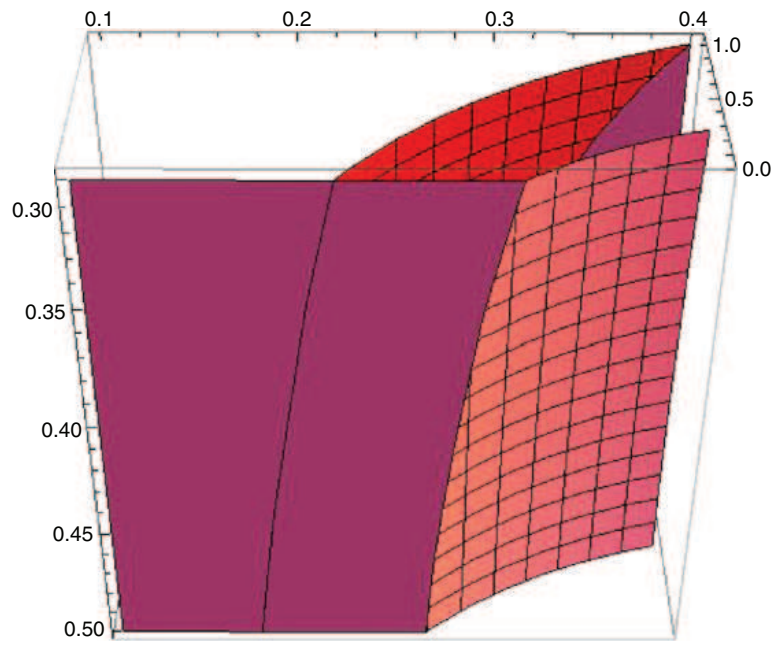

Fig. A.1. Graphical representation of $\mathrm{d} l / \mathrm{d} \phi$ (front) and $\mathrm{d} h / \mathrm{d} \phi$ (back) (Eqs. (A.2) and (A.3)) under $u=0.51$, and $l=0.61 * h$, for the year 2001 . $\phi$ on horizontal axis, $h$ on vertical axis. Values below zero are clipped.

\section{A.2. Partial derivatives for comparative statics}

$$
\begin{aligned}
& w_{h \phi}=\frac{A^{1-\alpha}(\alpha-1) k^{\alpha}(1-\phi)^{\sigma-1}(h-h u)^{\sigma}\left((h(\phi-1)(u-1))^{\sigma}+(l \phi u)^{\sigma}\right)^{-\frac{\alpha+2 \sigma-1}{\sigma}}\left((\alpha-1) \phi\left((h(\phi-1)(u-1))^{\sigma}+(l \phi u)^{\sigma}\right)-(\alpha+\sigma-1)(l \phi u)^{\sigma}\right)}{h \phi(u-1)} \\
& w_{h h}=-\frac{A^{1-\alpha}(\alpha-1) k^{\alpha}(1-\phi)^{\sigma}(h-h u)^{\sigma}\left((h(\phi-1)(u-1))^{\sigma}+(l \phi u)^{\sigma}\right)^{-\frac{\alpha+2 \sigma-1}{\sigma}}\left(\alpha(h(\phi-1)(u-1))^{\sigma}-(\sigma-1)(l \phi u)^{\sigma}\right)}{h^{2}(u-1)} \\
& w_{h l}=-\frac{A^{1-\alpha}(\alpha-1) k^{\alpha}(1-\phi)^{\sigma}(\alpha+\sigma-1)(l \phi u)^{\sigma}(h-h u)^{\sigma}\left((h(\phi-1)(u-1))^{\sigma}+(l \phi u)^{\sigma}\right)^{-\frac{\alpha+2 \sigma-1}{\sigma}}}{h l(u-1)} \\
& w_{h k}=\frac{A^{1-\alpha}(\alpha-1) \alpha k^{\alpha-1}(1-\phi)^{\sigma}(h-h u)^{\sigma}\left((h(\phi-1)(u-1))^{\sigma}+(l \phi u)^{\sigma}\right)^{-\frac{\alpha+\sigma-1}{\sigma}}}{h(u-1)} \\
& r_{\phi}=-\frac{A^{1-\alpha}(\alpha-1) \alpha k^{\alpha-1}\left((h(\phi-1)(u-1))^{\sigma}+(l \phi u)^{\sigma}\right)^{-\frac{\alpha+\sigma-1}{\sigma}}\left(\phi(h(\phi-1)(u-1))^{\sigma}+(\phi-1)(l \phi u)^{\sigma}\right)}{(\phi-1) \phi} \\
& r_{l}=-\frac{A^{1-\alpha}(\alpha-1) \alpha k^{\alpha-1}(l \phi u)^{\sigma}\left((h(\phi-1)(u-1))^{\sigma}+(l \phi u)^{\sigma}\right)^{-\frac{\alpha+\sigma-1}{\sigma}}}{l} \\
& r_{h}=-\frac{A^{1-\alpha}(\alpha-1) \alpha k^{\alpha-1}(h(\phi-1)(u-1))^{\sigma}\left((h(\phi-1)(u-1))^{\sigma}+(l \phi u)^{\sigma}\right)^{-\frac{\alpha+\sigma-1}{\sigma}}}{h} \\
& r_{k}=A^{1-\alpha}(\alpha-1) \alpha k^{\alpha-2}\left((h(\phi-1)(u-1))^{\sigma}+(l \phi u)^{\sigma}\right)^{\frac{1-\alpha}{\sigma}} \\
& S_{h}=-\frac{\left(\frac{h}{l}\right)^{\sigma} l((h-1) \sigma+1)}{h^{2}(l-1)} \\
& S_{l}=\frac{(h-1)\left(\frac{h}{l}\right)^{\sigma}((l-1) \sigma+1)}{h(l-1)^{2}} \\
& S_{\phi}=-\frac{\left(\frac{1}{\phi}-1\right)^{-\sigma}(\psi-1) \sigma\left(\frac{1}{u}-1\right)^{-\sigma}(u-1)}{(\phi-1) \phi \psi u} .
\end{aligned}
$$

\section{References}

Acemoglu, D., 2002. Technical Change, Inequality, and the Labor Market. Journal of Economic Literature 40 (1), 7-72.

Autor, D.H., Katz, L.F., Krueger, A.B., 1998. Computing Inequality: Have Computers Changed the Labor Market?. Quarterly Journal of Economics 113 (4), 1169-1213.

Benhabib, J., Farmer, R.E.A., 1994. Indeterminacy and Increasing Returns. Journal of Economic Theory 63, 19-41.

Benhabib, J., Perli, R., 1994. Uniqueness and Indeterminacy: On the Dynamics of Endogenous Growth. Journal of Economic Theory 63, 113-142.

Berman, E.J., Bound, J., Machin, S., 1998. Implications of skill-biased technological change: International evidence. Quarterly Journal of Economics 113, 1245-1280.

Burda, M.C., Severgnini, B., 2009. TFP Growth in Old and New Europe. Comparative Economic Studies 51 (4), 447-466. 
Caselli, F., Coleman, W.J., 2006. The World Technology Frontier. The American Economic Revue 96 (3), 499-521.

Cieślik, A., 2008. Multinational Firms, International Knowledge Flows, and Dual Labor Markets in Developing Economies. Review of Development Economics $12(1), 160-179$.

Esposito, P., Steher, R., (2007). The sector bias of skill-biased technical change and the rising skill premium in transition economies. wiiw Working Papers 43.

Galor, O., Moav, O., 2000. Ability Biased Technological Transition, Wage Inequality and Economic Growth. Quarterly Journal of Economics 115, 469-498. International Labor Organization (2008). Labor Statistics, Geneva.

King, R.G., Rebelo, S., 1999. Resuscitating Real Business Cycles. In: Taylor, J.B., Woodford, M. (Eds.), Handbook of Macroeconomics. Elsevier.

Lorentowicz, A., Marin, D., Raubold, A., (2005). Is human capital losing from outsourcing? Evidence from Poland and Austria. CESifo Working Paper 1616. Machin, S., 2008. An Appraisal of Economic Research on Changes in Wage Inequality. LABOUR 22 (S1), 7-26.

Machin, S., Van Reenen, J., 1998. Technology and changes in skill structure: evidence from seven OECD countries. Quarterly Journal of Economics 113 $1215-1244$.

Mortensen, D.T., Pissarides, C.A., 1999. New developments in models of search in the labor market. In: Oshenfelter, A., Card, D. (Eds.), Handbook of Labor Economics. Elsevier, pp. 2567-2627.

Polish Central Statistical Office (2007). Earnings of the employes in the national economy by occupations as of March 1996; Earnings by occupations in October 1998; Structure of wages and salaries by occupation in October: 1999, 2001, 2002, 2004, 2006; Poland. Polish Central Statistical Office GUS.

Prasad, E., 1996. Skill heterogeneity and the business cycle. Canadian Journal of Economics 29 (4), 910-929.

Prescott, E.C., 2004. Why Do Americans Work So Much More Than Europeans. Federal Bank of Minneapolis Quarterly Review 28 (1), 2-13.

Stokey, N., Rebelo, S., 1995. Growth effects of flat-rate taxes. Journal of Political Economy 103 (3), 519-550.

US Bureau of Labour Statistics (2008). US Bureau of Labour Statistics. 